\title{
Genome-wide analysis of polygalacturonase gene family from pear genome and identification of the member involved in pear softening
}

Suling Zhang ${ }^{1 \dagger}$, Min Ma ${ }^{1 \dagger}$, Huping Zhang ${ }^{1 \dagger}$, Shaoling Zhang ${ }^{1}$, Ming Qian ${ }^{1}$, Zhen Zhang ${ }^{1}$, Weiqi Luo ${ }^{2}$, Jinbu Fan ${ }^{1}$, Zhiqiang Liu ${ }^{1}$ and Libin Wang ${ }^{1 *}$

\begin{abstract}
Background: Polygalacturonase (PG), as an important hydrolase participating in the degradation of pectin, plays an important role in softening process of fruit. However, information on $P G$ gene family in pear genome and the specific member involved in fruit softening is still rudimentary.

Results: In this study, a total of 61 PG genes, which could be divided into six subclasses, were identified from the pear genome with diverse chromosome locations, gene structures, motifs and cis-acting elements. Most PbrPGs were derived from WGD/segmental duplication blocks, and purifying selection was the main driving force for their expansion. The expression profiles of PbrPGs in pear were tissue/development-stage/cultivar-dependent. During 'Housui' pear storage, associated with the reduction of firmness was the accumulation of PG activity. Totally, 28 PbrPGs were expressed during fruit storage, which could be classified into five categories based on different expression patterns; most demonstrated an increased trend. Of these, PbrPG6 were proposed to account for pear softening in combination of the phylogenetic and correlation analysis among firmness, PG activity and PbrPGs. By constructing the silencing vector, a higher firmness was observed in PbrPG6-silenced fruit when compared with that of the control (empty vector). In a further study, we found that the expression of PbrPG6 was regulated by postharvest 1-MCP/ethrel treatment, and several PbrERFs might function in this process.

Conclusions: We identified 61 PbrPG genes from pear genome; of these, PbrPG6 was involved in fruit softening process; furthermore, the expression of PbrPG6 might be under the control of PbrERF. This study provides a foundation for future work aimed at elucidating the molecular mechanism underlying pear softening.
\end{abstract}

Keywords: Polygalacturonase, Gene family, Pear fruit, Firmness, Ethylene response factor

\section{Background}

Ripening \& senescence of horticultural fruit is a very complex process, which accompanies with the changes in color, texture and flavor [1]. As one of the most obvious phenomena, the reduction of firmness during storage could enhance the sensitivity of fruit to mechanical damage and thus shorten their shelf life [2]. Fruit

\footnotetext{
* Correspondence: wanglibin@njau.edu.cn

†'Suling Zhang, Min Ma and Huping Zhang contributed equally to this work. ${ }^{1}$ Centre of Pear Engineering Technology Research, State Key Laboratory of Crop Genetics and Germplasm Enhancement, Nanjing Agricultural University, Nanjing 210095, Jiangsu, China

Full list of author information is available at the end of the article
}

softening is mainly due to the alternation in cell wall structure and composition, including cellulose, hemicellulose, and pectin [3]. Pectin, a major component of the primary cell wall, play a critical role in cellular structural integrity and cell adhesion [4].

Belonging to one of the largest hydrolase families, polygalacturonase (PG), which was discovered half a century ago, has known to be involved in various processes of plant development, such as flower development, fruit ripening \& senescence and organ abscission [5-7]. PG plays an important role in pectin disassembly, and could be divided into three types based on different

(C) The Author(s). 2019 Open Access This article is distributed under the terms of the Creative Commons Attribution 4.0 International License (http://creativecommons.org/licenses/by/4.0/), which permits unrestricted use, distribution, and 
catalytic processes, including endo-PGs, exo-PGs, and rhamno-PGs [6]. Until recently, $P G$ family genes have been identified from various plants, such as Arabidopsis, Oryza sativa, Brassica rapa, Populus, cucumber, watermelon, tomato, mango, apple and peach [6-8]. Eleven members from Populus were proposed to be involved in flower development, while two related to leaf abscission under salt stress [9]. Of 54 SlPGs identified from tomato fruit, members in clade A and B were involved in fruit and abscission zone development, while members from clade $\mathrm{C}, \mathrm{D}$, and $\mathrm{F}$ in flowering development [7]. Three PpPGs supposedly participated in the softening process of peach [8]. These results implied that there was extensive functional differentiation among plant $P G$ genes.

Ethylene plays an important role in the ripening \& senescence process of climacteric fruit [10]. Mutation of an ethylene receptor, Never-ripe ( $\mathrm{Nr}$ ), suppressed the ripening process of tomato fruit; furthermore, the expression of $37 \%$ genes was altered in transgenic fruit, causing distinct seed number, ascorbate \& carotenoid abundance [11]. As the final response gene in the ethylene signaling pathway, ethylene response factors (ERFs) trigger an ethylene response and regulate fruit ripening by binding to the promoters of several ripening-related genes, such as pectin methylesterase $(P M E)$, 1-aminocyclopropane-1-carboxylic acid oxidase $(A C O)$, and $P G[10,12,13]$. Electrophoretic mobility shift assay (EMSA) demonstrated a specific binding of CPERF9 to the promoters of CpPG5 and CPPME1/2 in papaya, via the GCC-box motif [13]. A majority of ERFs would activate the transcription of ripeningrelated genes, while some demonstrated a reverse impact [10].

Pear, as a respiratory climacteric fruit, is popular because of its juicy and delicious taste $[14,15]$. During fruit storage, associated with the change in the composition of flavor contributors was the reduction of firmness [14, 16-18], which PG might played an important role in [19]. However, our knowledge on pear $P G$ gene family and the specific member involved in fruit softening was still rudimentary. In this study, the identification of $P G$ genes from pear genome was performed to analyze their chromosome localizations, gene structures, motif composition as well as cis-acting elements. Their expression profiles in different tissues as well as during fruit development/storage were determined. In combination with the results of phylogenetic and correlation analysis among firmness, PG activity and PbrPGs transcripts, member playing an important role during the softening process of pear fruit was identified and functionally validated through the construction of transient silencing vector. Furthermore, the candidate ERFs possibly involved in regulating the expression of the key $P G$ gene were summarized.

\section{Result}

\section{Identification and phylogenetic analysis of PbrPGs}

Totally, $61 P G$ genes were identified from pear genome, which were named as PbrPG1-61 based on their chromosomal location (Additional file 2: Table S1). Of these, 43 genes contained the conserved domains I, II, III and IV; PbrPG18 and 52 lacked the domain I; 14 members did not possess the domain III; PbrPG2 and 3 lacked the domain IV; PbrPG8 lacked the domain II and III (Additional file 1: Figure S1 and Additional file 2: Table S1). Eighty ESTs hits were identified for all PbrPGs with the greatest number for PbrPG7, 46, 57, and 58 (Additional file 2: Table S2).

Referring to the biological classification of $P G s$ from peach and Arabidopsis, PbrPGs could be grouped into six subclasses (subclasses A to F), including 8 (A), 6 (B), 7 (C), 20 (D), 12 (E) and 8 (F) members, respectively (Fig. 1a). Subclass $G$ was composed of three $P G$ genes from Arabidopsis, excluding any members from pear and peach (Fig. 1a). Besides, PbrPG48, 55, 31, 56, 23, 20, 21, 22, 24 and PbrPG9, 34, 28, 52 in subclass D constituted two special subgroups without members from other species (Fig. 1a).

The length, molecular weight, isoelectric point $(p I)$, extinction coefficient, instability index, aliphatic index and grand average of hydropathicity (GRAVY) of PbrPGs were within the ranges of 234-753 amino acids, 24.74$82.23 \mathrm{kDa}, \quad 4.61-9.70, \quad 10,345-88,975, \quad 23.39-61.89$, 28.53-97.47, - 0.366-0.109, respectively (Additional file 2: Table S1). Instability index is used to determine whether the protein is stable in a test tube $(\leq 40$, probably stable; $>40$, probably not stable) [15]. Thus, most PbrPGs were predicted to be stable (Additional file 2: Table S1). GRAVY values for most PbrPGs were below zero, suggesting that they were hydrophilic (Additional file 2: Table S1). SignalP 4.1 analysis revealed that PbrPG1-3, 5-7, 12-16, 18, 20-27, 29, 33-40, 42, 43, 45, 47, 49-51, 53-58 contained signal peptides (Additional file 2: Table S1).

\section{Gene and protein feature of PbrPGs}

As shown in Fig. 1b, the number of exons (intron) in PbrPGs ranged from 1 (0) to 11 (10); members in subclasses A, B and F generally possessed more exons/introns than others; in addition, the exon/intron structure in the same subclass was relatively conserved. Furthermore, the average intron/exon number of PbrPGs were higher/larger than those in the whole genome; and the average GC3 percentage of PbrPGs was lower than the average level in the whole genome (Additional file 2: Table S3).

One hundred five cis-acting elements were identified from the promoters of PbrPGs, which could be divided into eight categories (Additional file 2: Table S4), 


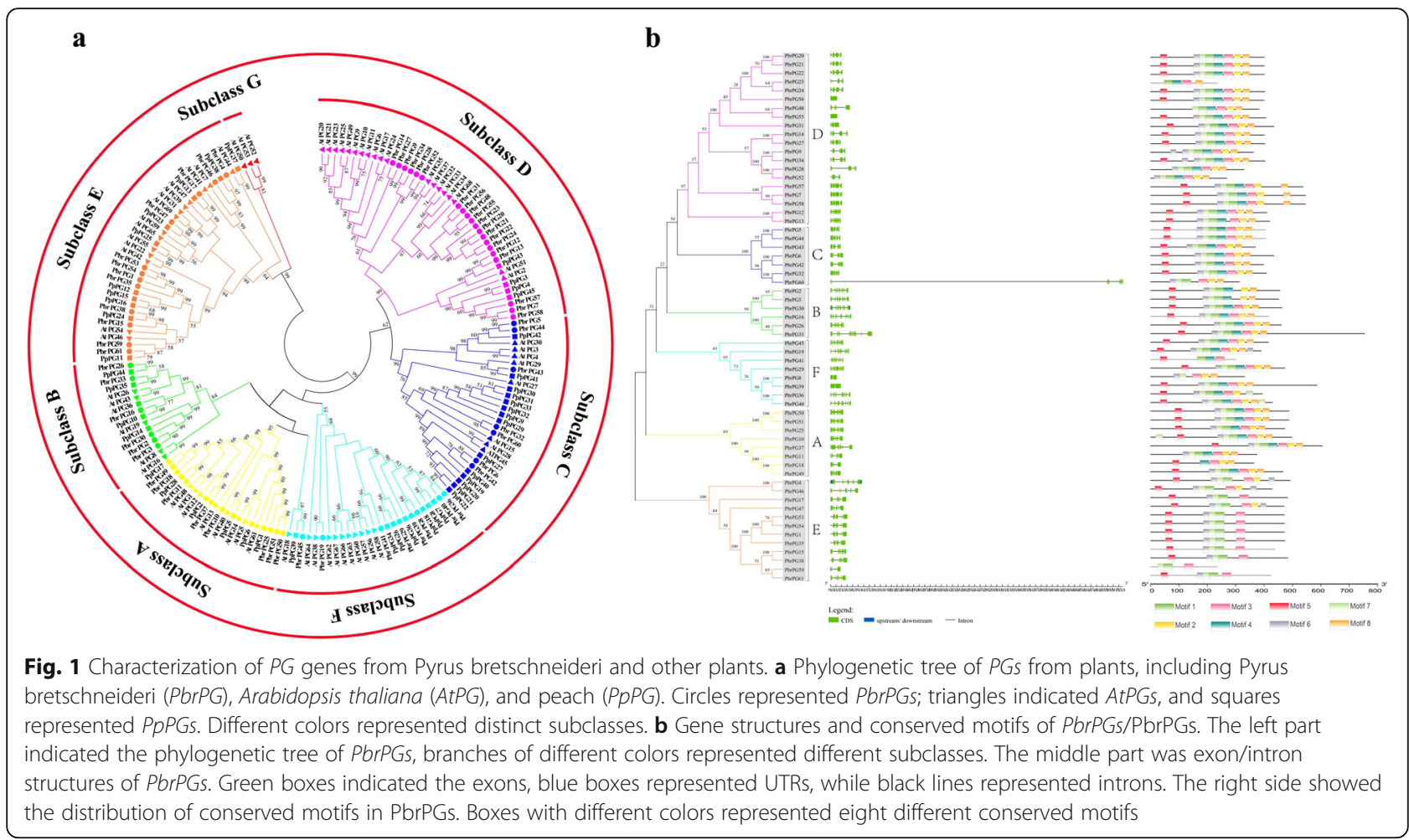

including conserved promoter motifs $(n=2)$, light responsive elements $(n=26)$, phytohormone responsive elements $(n=12)$, defense/abiotic stress responsive elements $(n=5)$, tissue/organelle specific elements $(n=$ $7)$, pathogen/elicitor/wound responsive elements $(n=1)$, miscellaneous elements $(n=10)$ and elements with unknown functions $(n=42)$ [15]. Their distribution in
PbrPGs were distinct (Additional file 2: Table S4). Comparative analysis of upstream regions of close paralogs showed divergence in the promoters of duplicated genes (Additional file 1: Figure S2).

Eight motifs were found in PbrPGs, with diverse distributions (Additional file 2: Table S5): 96.7\% members contained motif 1 and 3, while several motifs only

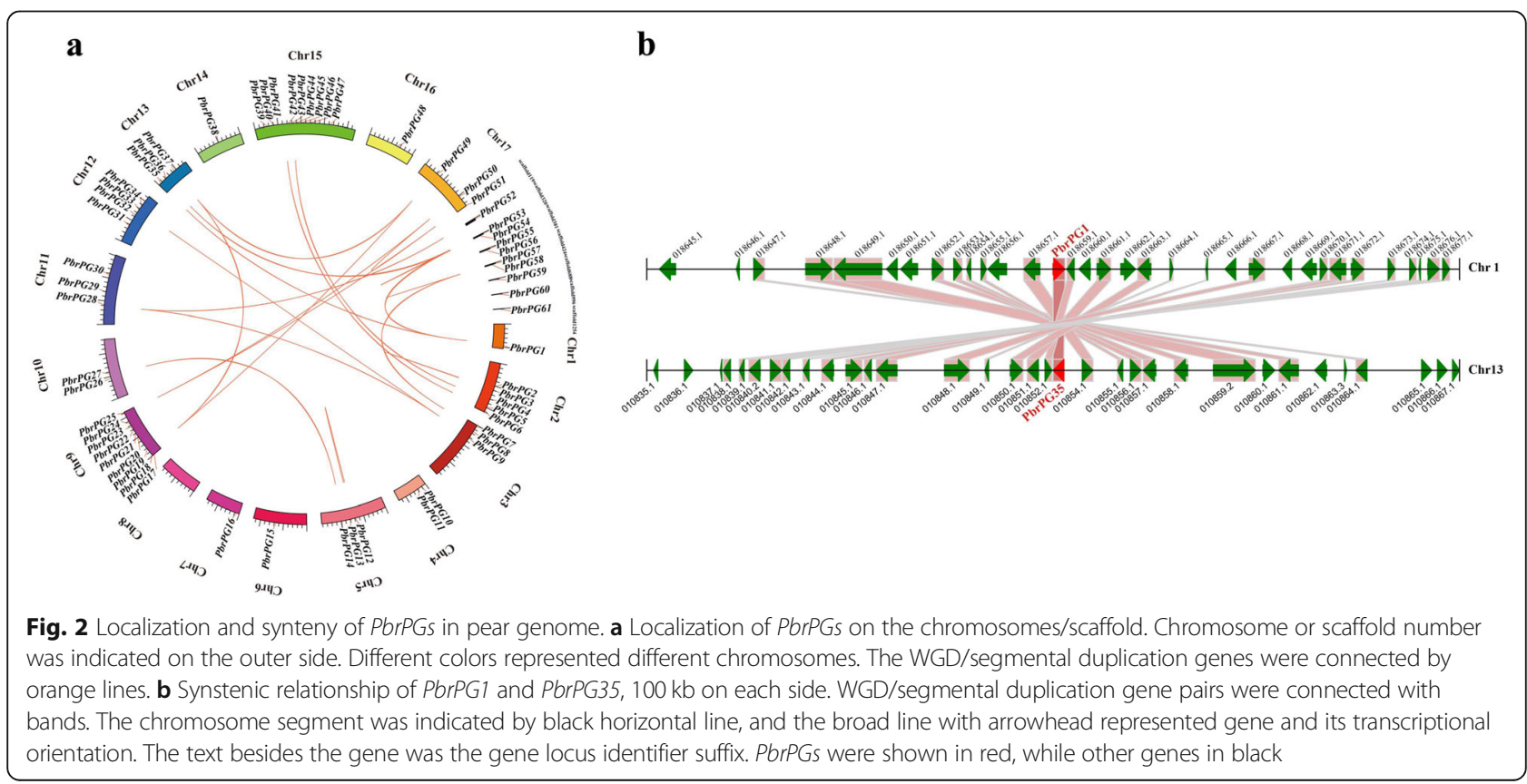


existed in certain subclasses (for example, subclass E did not possess motif 2,4 and 8 ).

\section{Chromosomal location, gene duplication and $\mathrm{Ka} / \mathrm{Ks}$ analysis}

Most PbrPGs were distributed on 16 chromosomes with an uneven distribution (Fig. 2a). Twenty-six genes were derived from WGD/segmental duplication; 22 and 12 members were assigned to dispersed and tandem duplication block, respectively; on the other hand, only $1.64 \%$ was derived from proximal duplication (Additional file 2: Table S6). An all-vs.-all local BLASTP based on a method similar to the one used for PGDD was performed across the whole pear genome to identify synteny blocks. Conserved synteny was observed in the regions containing PbrPGs. Take PbrPG1 \& PbrPG35, highly conserved synteny was observed in the regions containing these genes (Fig. 2b).

As shown in Additional file 2: Table S7, seven duplicated gene pairs have similar Ks values (0.159-0.237), suggesting that they might be derived from the recent WGD/segmental duplication (30-45 MYA); 11 gene pairs had smaller Ks values (0-0.141), suggesting that they may come from more recent WGD/segmental duplication; PbrPG5 \& PbrPG42 (Ks 2.000) might arise from the $\gamma$ triplication ( $140 \mathrm{MYA})$ [20]. Moreover, the $\mathrm{Ka} / \mathrm{Ks}$ ratios of 15 paralogous gene pairs were less than one, implying that purifying selection was the primary driving force for PbrPGs [15].

\section{Expression profiles of PbrPGs in different tissues and during pear fruit development}

The expression profiles of PbrPGs in different tissues of 'Yali' pear were distinct, with the highest total abundance in stigma and lowest in petal. 34, 24, 26, 25, 32 and 23 genes were expressed in stigma, shoot, ovary, leaf, petal and 15 DAFB fruit, respectively; and the abundance of PbrPG61 mRNAs was highest in fruit, petal, leaf and ovary, while PbrPG4 and PbrPG35 demonstrated the highest expression in shoot and stigma, respectively (Fig. 3a).

Twenty-one PbrPGs were transcribed during 'Housui' fruit development with diverse expression patterns: PbrPG6, 35 and 42 mRNAs showed an increased trend throughout fruit maturity, while $\operatorname{PbrPG37}$ and 38 decreased; the expression of PbrPG4, 33 and 46 was downregulated during early stage before an increase, while a opposite phenomenon was observed for PbrPG2, 13, 17, $37,47,49,55$ and 61 ; on the other hand, the transcript abundance of other members fluctuated (Fig. 3b) [21]. Moreover, the expression profiles of PbrPGs during fruit development were cultivar-dependent (Additional file 1: Figure S3) [21].

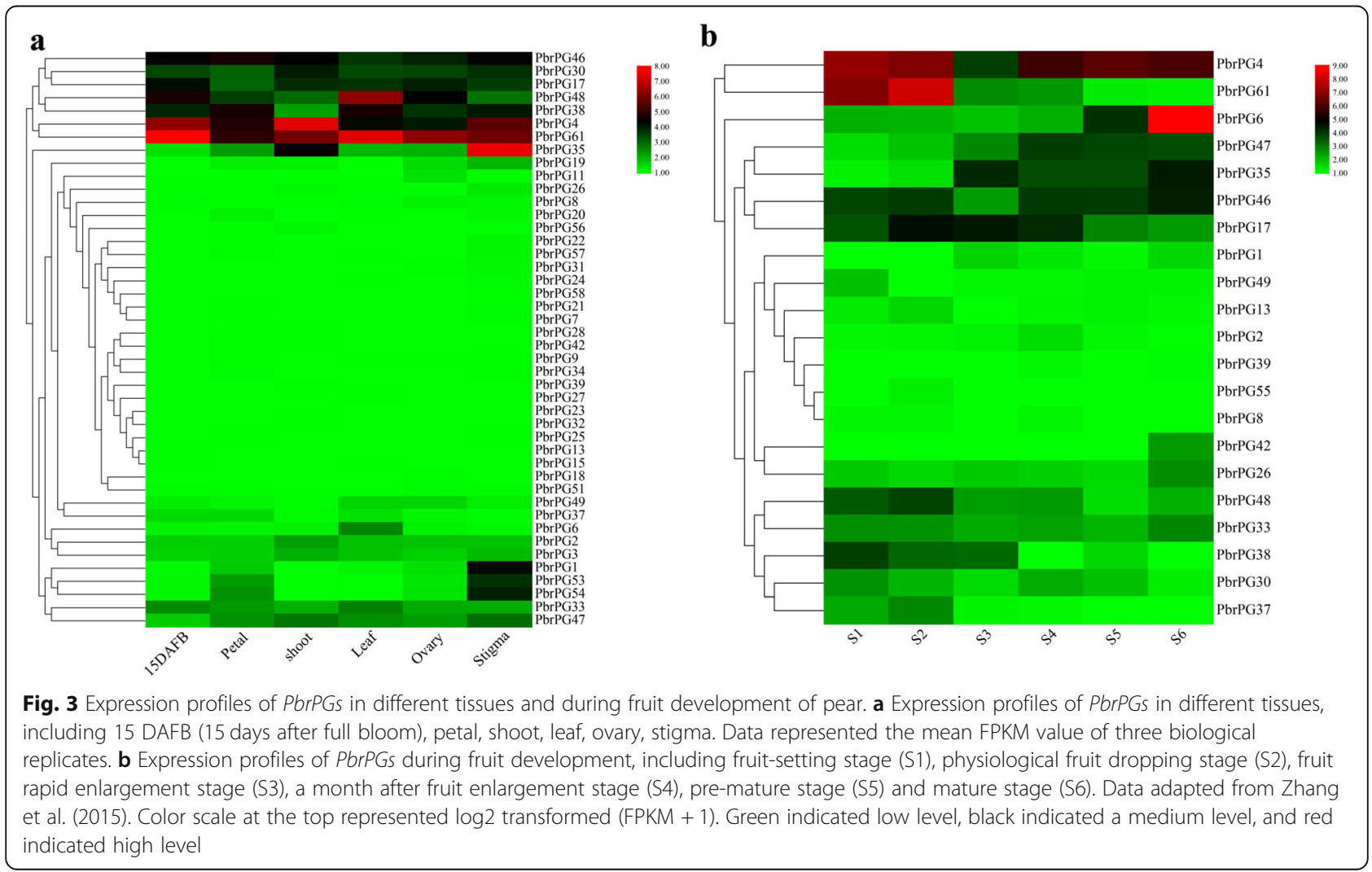




\section{Identification of PbrPGs involved in pear softening}

During 'Housui' pear storage, a value in pericarp, weight loss and decay rate steadily accumulated, while firmness in fruit cortex decreased, which was associated with the alternation of ethylene evolution and respiration rateboth increased from 0th $\mathrm{d}$ to 18 th $\mathrm{d}$, and then decreased (Additional file 1: Figure S4 and Table 1). For PG activity, it accumulated throughout storage, with a $52 \%$ increment (Fig. 4a).

Twenty-eight out of sixty-one PbrPGs were expressed during postharvest storage of 'Housui' pear; and the transcripts of 14 members could be detected at every stage (Fig. 4b). Based on the distinct expression patterns, they could be divided into five groups: mRNA abundances of genes in Group I, including PbrPG6, 10, 14, $26,31,35,39,43$ and 47 , showed an increased trend throughout storage; the expression of PbrPG3, 4, 17 and 30 in Group II was downregulated; the transcription of members in Group III, such as PbrPG2, 46, 49 and 51, was inhibited at the early stages before a increment; the expression pattern of genes (PbrPG1, 33, 37, 48, 52, 53, 54) in Group IV was opposite to that in Group III; on the other hand, the expression of other members in Group V fluctuated during storage. RT-qPCR analysis validated the accuracy of transcriptome data on the expression patterns of PbrPGs (Fig. 4b and Additional file 1: Figure S5a).

Correlation analysis among PbrPGs mRNAs, PG activity, and firmness were performed, and genes with high correlation coefficients with PG activity as well as firmness (> 70\%) were summarized, including PbrPG4, 6, 17, 26, 46 and 61 (Fig. 4c). Of these, PbrPG4, 6, 17, 46 and 61 were cluster with PpPG15, 21 and 22 (Fig. 1a), which were proposedly involved in the softening process of peach fruit [8]; meanwhile, PbrPG6 and 26 demonstrated a similar pattern as PpPG15, 21 and 22 during storage (Fig. $4 \mathrm{~b}$ and [8]). These results implied that PbrPG6 might play a key role in fruit softening process.

In order to validate its function, we then constructed the silencing vector for transient transformation of pear fruit. As shown in Fig. 5, a higher firmness was observed for the cortex of transgenic fruit with lower level of PbrPG6 mRNAs and PG activity, when compared with that of the control.

\section{Candidate PbrERFs regulating the expression of PbrPG6}

As shown in Fig. 4c, a positive correlationship was observed between PbrPG6 mRNAs and ethylene evolution. Besides, an opposite impact on PbrPG6 expression, PG activity and cortex firmness was observed after 1-MCP and ethrel treatment of pear fruit: 1-MCP fumigation suppressed PbrPG6 mRNAs and PG activity, resulting in a higher cortex firmness when compared with that of the control; on the other hand, PbrPG6 expression and PG activity were upregulated by ethrel dipping, and cortex firmness of ethrel-treated fruit was lower than control (Fig. 6). A similar result was observed in a previous study conducted in out unit in 2017 (Additional file 1: Figure S6). Besides, fruit with upregulated expression of PbrACO1, which could enhance ethylene evolution [15], demonstrated higher abundances of PbrPG6 mRNAs as well as lower firmness (Additional file 1: Figure S7). Furthermore, PbrPG6 contained GCC-box within $2000 \mathrm{bp}$ upstream from the translational starting site (https:// www.dna.affrc.go.jp/PLACE/) (Additional file 2: Table S8) [22]. These results suggested that the expression of PbrPG6 might be regulated by ethylene, and PbrERF might participate in this process.

Based on previous report on ERF family genes in pear genome [23] as well as transcriptome analysis, 100 out

Table 1 Quality changes during 'Housui' pear storage ${ }^{\text {a }}$

\begin{tabular}{|c|c|c|c|c|c|c|}
\hline \multirow[t]{2}{*}{ Attribute } & & \multicolumn{5}{|l|}{ Storage time/d } \\
\hline & & 0 & 6 & 12 & 18 & 24 \\
\hline Weight loss (\%) & & $0.00 \pm 0.00^{b}$ & $0.72 \pm 0.19$ & $1.45 \pm 0.21$ & $2.35 \pm 0.39$ & $3.10 \pm 0.46$ \\
\hline Firmness (N) & & $254.00 \pm 12.17$ & $223.67 \pm 6.66$ & $131.33 \pm 21.46$ & $107.33 \pm 4.93$ & $95.33 \pm 3.06$ \\
\hline Decay rate (\%) & & $0.00 \pm 0.00$ & $0.00 \pm 0.00$ & $9.00 \pm 1.00$ & $17.00 \pm 1.00$ & $22.00 \pm 2.00$ \\
\hline \multirow[t]{3}{*}{ Color (Pericarp) } & $L^{*}$ & $55.41 \pm 0.34$ & $54.19 \pm 1.79$ & $55.42 \pm 0.55$ & $54.73 \pm 1.30$ & $55.85 \pm 2.58$ \\
\hline & $a^{*}$ & $1.98 \pm 0.21$ & $2.22 \pm 0.58$ & $5.05 \pm 1.56$ & $8.80 \pm 1.12$ & $9.68 \pm 1.56$ \\
\hline & $b^{*}$ & $31.97 \pm 0.35$ & $34.00 \pm 1.89$ & $33.80 \pm 0.74$ & $34.25 \pm 0.82$ & $34.94 \pm 3.28$ \\
\hline \multirow[t]{3}{*}{ Color (Cortex) } & $L^{*}$ & $66.24 \pm 2.45$ & $58.73 \pm 2.97$ & $61.23 \pm 2.64$ & $60.05 \pm 3.19$ & $66.00 \pm 5.60$ \\
\hline & $a^{*}$ & $-1.46 \pm 0.11$ & $-1.52 \pm 0.13$ & $-1.51 \pm 0.19$ & $-1.64 \pm 0.14$ & $-1.53 \pm 0.09$ \\
\hline & $b^{*}$ & $8.08 \pm 0.34$ & $6.89 \pm 0.49$ & $7.96 \pm 0.70$ & $8.20 \pm 0.43$ & $7.86 \pm 0.99$ \\
\hline
\end{tabular}

${ }^{a}$ Uniform and defect-free 'Housui' pear fruit were selected, randomly divided into several groups, packed with plastic bags, and then stored at $25^{\circ} \mathrm{C}$. Samples were taken every $6 \mathrm{~d}$ until decay rate over $20 \%$

${ }^{b}$ Data represented the mean value \pm SE of three biological replicates

The symbol ${ }^{*}$ reflects the color measurment description. It is a standard color measurment notation. 

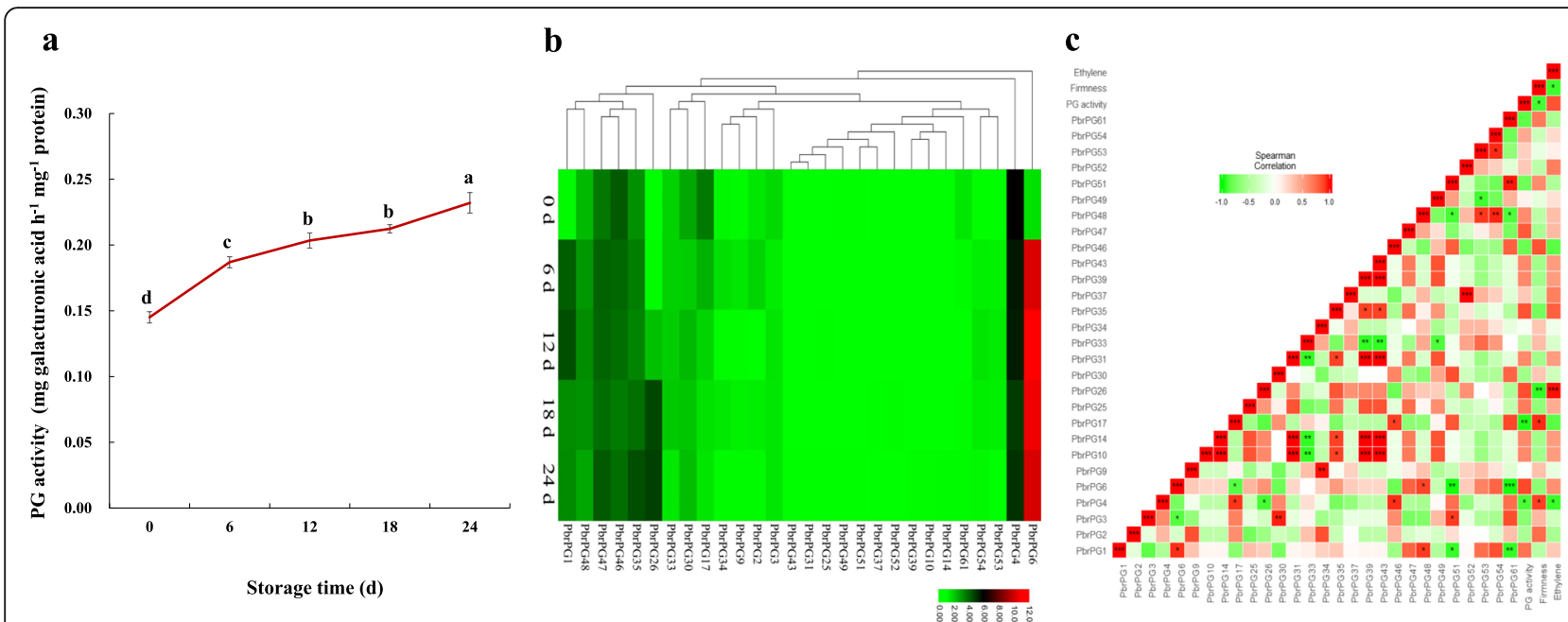

Fig. 4 Dynamic changes of PG activity and PbrPG mRNAs during 'Housui' pear storage. a Dynamic change of PG activity. Data represented the mean value \pm SE of three biological replicates. Different small letters with the same treatment mean significant difference among samples $(p<0.05)$. $\mathbf{b}$ Heatmap of the expression profiles of PbrPGs. Uniform and defect-free 'Housui' pear fruit were selected, randomly divided into several groups, packed with plastic bags, and then stored at $25^{\circ} \mathrm{C}$. Samples were taken every $6 \mathrm{~d}$ until decay rate over 20\%. Color scale at the top represented log2 transformed (FPKM +1$)$. Green indicated low level, black indicated a medium level, and red indicated high level. c Correlation coefficients among firmness, ethylene evolution, PG activity and PbrPG mRNAs. Pearson correlations between attributes were visualized as a heat map, where negative correlations are represented in green and positive correlations in red

of 155 PbrERFs were expressed during 'Housui' pear storage with diverse expression patterns (Fig. 7a). RTqPCR analysis validated the accuracy of transcriptome data on the expression patterns of PbrERFs (Additional file 1: Figure S5b). Correlation analysis found that 33 members illustrated high correlation coefficients $(>70 \%)$ with PbrPG6. Of these, Pbr1ERF5/6, Pbr3ERF21, Pbr4ERF24, Pbr5ERF28, Pbr12ERF100, Pbr13ERF110, Pbr15ERF126/129/136, and Pbr17ERF148 were positively correlated with PbrPG6; on the other hand, a negative relationship was observed between PbrPG6 and other PbrERFs (Pbr1ERF1/2, Pbr3ERF15/19/20, Pbr4ERF26, Pbr5ERF38/39, Pbr6ERF40/45/48/51, Pbr8ERF67,

\section{Pbr9ERF74/76, Pbr1OERF80/84, Pbr11ERF86/90,} Pbr13ERF106/107, and Pbr15ERF138) (Fig. 7b).

Besides, 30 members illustrated high correlation coefficients (>70\%) with ethylene (Fig. 7b). Of these, Pbr2ERF13, Pbr3ERF17, Pbr5ERF28, Pbr7ERF61, Pbr9ERF72/73, Pbr1OERF78/85, Pbr11ERF87, Pbr12ERF95-97, Pbr13ERF110, Pbr14ERF119, Pbr15ERF133-136, Pbr16ERF144/146, and Pbr17ERF148 were positively correlated with ethylene. On the other hand, a negative relationship was observed between ethylene and other PbrERFs, including Pbr1ERF8, Pbr5ERF30/ 31, Pbr6ERF51, Pbr10ERF77, Pbr13ERF105/107,
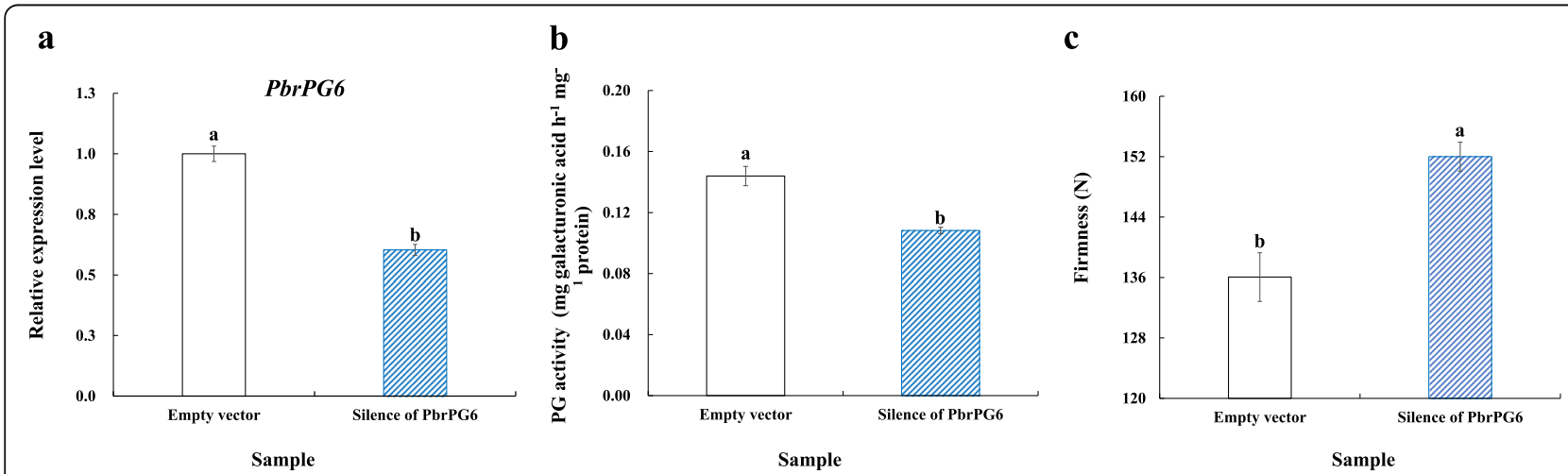

Fig. 5 Impact of transient silence of PbrPG6 expression on PG activity and firmness of pear fruit. a Expression profile of PbrPG6 after transient silence of PbrPG6 expression. $\mathbf{b}$ Impact of transient silence of PbrPG6 expression on PG activity. $\mathbf{c}$ Impact of transient silence of PbrPG6 expression on cortex firmness. 'Housui' fruit were infiltrated with pTRV2:PbrPG6 \& pTRV1, and then stored at $25^{\circ} \mathrm{C}$ before analysis. The unconstructed pTRV2 vector, which was co-injected with pTRV1, was used as control. Data represented the mean \pm SD of three biological replicates. The expression level of PbrPG6 in control fruit was set as 1.0. Vertical bars labeled with different small letters indicated significant difference between samples at $p<0.05$ level using Duncan's multiple range test 

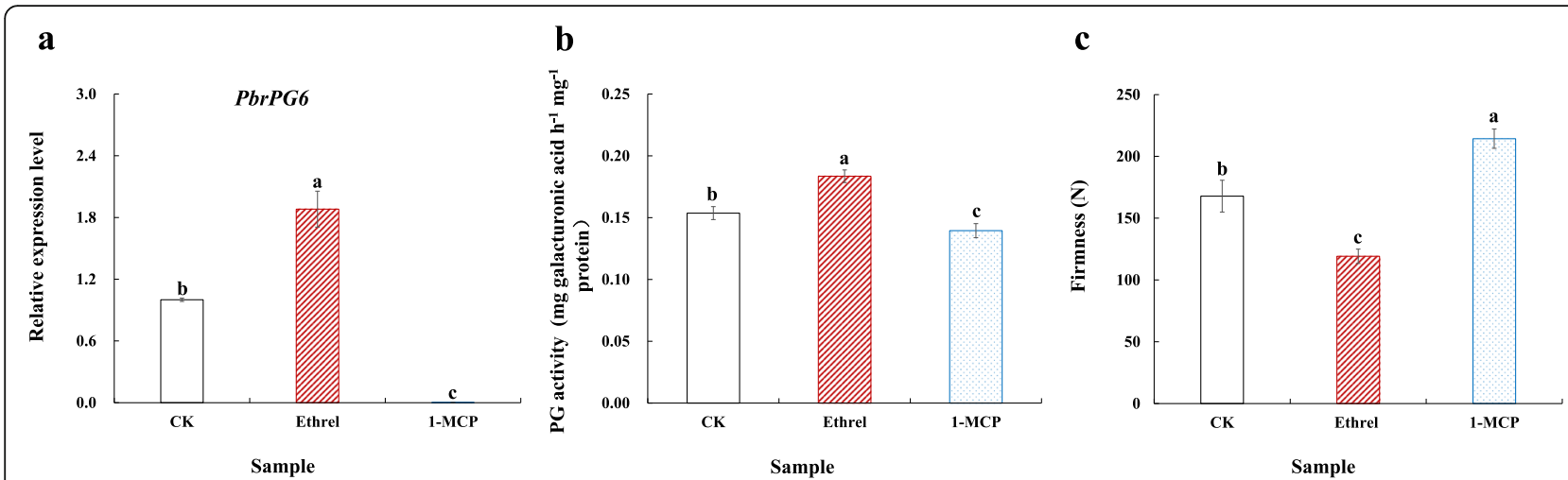

Fig. 6 Impact of 1-MCP/ethrel treatment on PbrPG6 expression, PG activity and cortex firmness of pear. a Expression profile of PbrPG6 after 1-MCP and ethrel treatments. $\mathbf{b}$ Impact of 1-MCP and ethrel treatments on PG activity. $\mathbf{c}$ Impact of 1-MCP and ethrel treatments on cortex firmness. 'Housui' pears, harvested from an experimental orchard in Nanjing, were divided into three treatments: (1) fumigated with $1.5 \mu \mathrm{LL}{ }^{-1} 1$-MCP for $24 \mathrm{~h}$, (2) dipped in $0.5 \mathrm{~mL} \mathrm{~L}^{-1}$ ethrel for $5 \mathrm{~min}$, and (3) dipped in $0.5 \mathrm{~mL} \mathrm{~L}^{-1} \mathrm{H}_{2} \mathrm{O}$ for $5 \mathrm{~min}$ (control). After treatments, fruits were packed with plastic bags and stored at $25^{\circ} \mathrm{C}$ for $5 \mathrm{~d}$. Data represent the mean \pm SE of three biological replicates. The expression level of PbrPG6 in control fruit was set as 1.0. Vertical bars labeled with different small letters indicated significant difference between samples at $p<0.05$ level using Duncan's multiple range test

\section{Discussion}

Pear, distributed on six continents with China as the leading producer, is popular among consumers for its unique flavor quality $[14,16]$. During 'Housui' pear storage, a" value in pericarp, weight loss and decay rate accumulated, while cortex firmness decreased (Table 1). Similar phenomenon was also observed for 'Yali' and 'Huanghua' pear during storage [14, 19].
Fruit softening, which is mainly due to the alternation in cell wall structure \& composition [3], could enhance the sensitivity of pear fruit to mechanical damage and thus shorten their shelf life [2]. Cell wall is consisted of complex polysaccharides, including pectin, cellulose, and hemicellulose [3]. A variety of enzymes, including PG, pectinesterase (PE), $\beta$ galactosidase $(\beta-\mathrm{Gal})$, cellulase and xyloglucan endo-
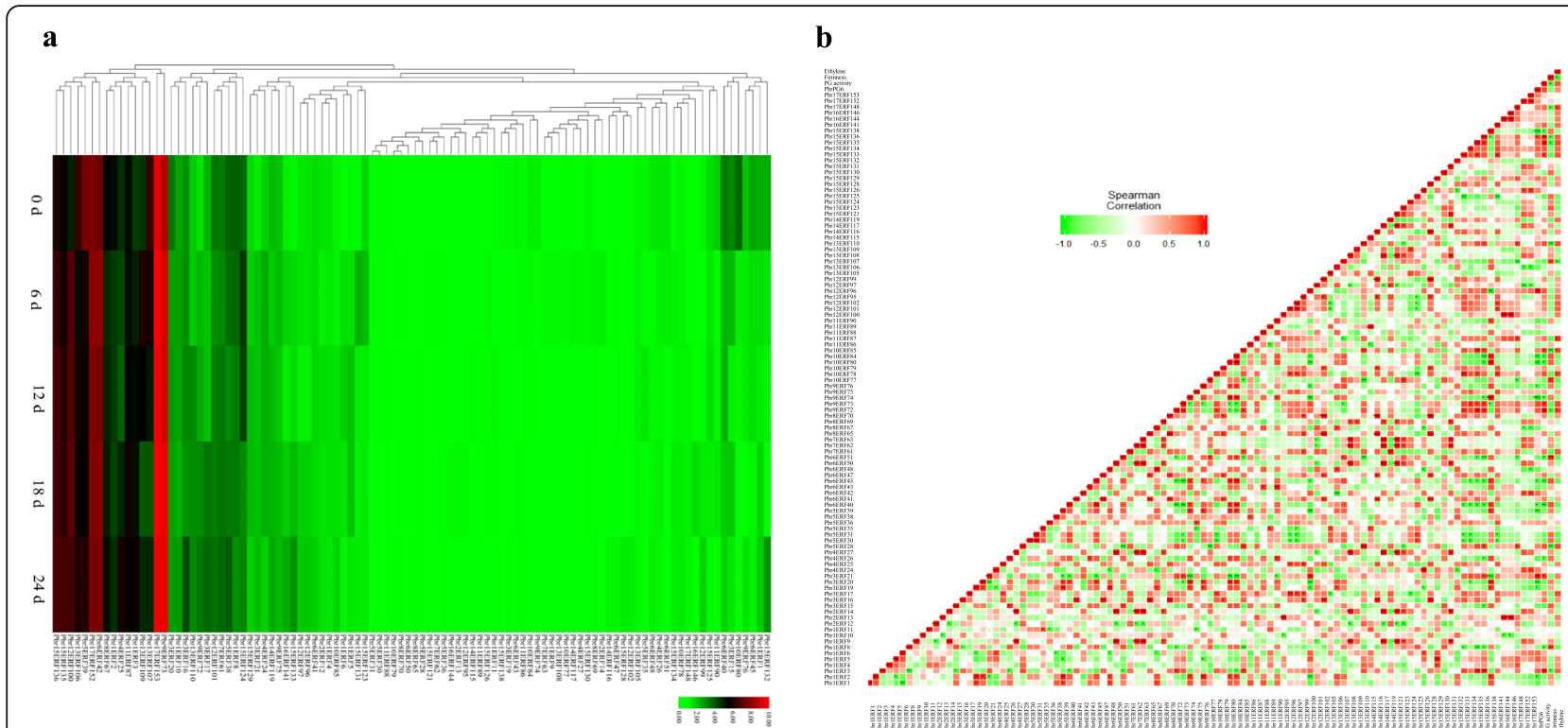

Fig. 7 Expression profiles of PbrERFs during 'Housui' pear storage. a Heatmap of the expression profiles of PbrERFs. Uniform and defect-free 'Housui' pear fruit were selected, randomly divided into several groups, packed with plastic bags, and then stored at $25^{\circ} \mathrm{C}$. Samples were taken every 6 d until decay rate over $20 \%$. Color scale at the top represented log2 transformed (FPKM + 1). Green indicated low level, black indicated a medium level, and red indicated high level. b Correlation coefficients among PbrERF transcripts, PG activity, ethylene evolution, and PbrPG6 mRNAs. Pearson correlation between attributes was visualized as a heat map, where negative correlations was represented in green and positive correlations in red 
transglycosylase (XET), are proposedly involved in the metabolism of these components [7, 24].

Pectin is a structural polysaccharide of the primary cell wall and middle lamella, playing an important role in cell-to-cell adhesion [7]. PG is the key enzyme involved in its degradation, through cleavage of $\alpha-(1 \rightarrow 4)$ glycoside bonds $[7,25]$. Until recently, $P G$ family genes have been characterized from many plants $[6,8,9,26]$. Besides fruit softening, PG also functions in many developmental processes of plant, such as flower development or abscission zone development [7]. During 'Huanghua' pear storage, firmness demonstrated an opposite trend when compared with that of PG activity [19]. A similar phenomenon was observed during 'Housui' fruit storage (Fig. 4a and Additional file 2: Table S1). However, our knowledge on $P G$ gene family in pear genome as well as their role in fruit softening is still rudimentary.

A total of 61 PbrPGs were identified from the pear genome with an uneven chromosomal distribution, which could be divided into six groups (A-F) (Fig. 1a). A similar result was observed in other plants [6-8]. In consistent with the previous report on PGs from in peach, most PbrPGs contained four conserved domains [8]: domains I \& II likely composed the catalytic site; domain III was involved in catalytic reaction; on the other hand, domain IV constituted a likely candidate for ionic interaction with carboxylate groups in the substrate [27].

The gene structures and the composition of motifs or cis-acting elements were distinct among PbrPGs/PbrPGs; members within the same class shared similar gene structure and components (Fig. 1b). Similar result were observed for other gene families in pear $[15,20]$. In agreement with the result of Wu et al. [28], most PbrPGs were assigned to WGD/segmental duplication, and the purifying selection was the primary driving force for their evolution (Additional file 2: Table S7). Polyploidy through WGD is frequently associated with genome rearrangement, and the evolution of genes is proposedly driven by a variety of factors, including structural complexity, conserved domain, and evolutionary rate [15]. In our study, PbrPGs possessed four highly-conserved domains (Additional file 1: Figure S1), and demonstrated lower $\mathrm{Ka} / \mathrm{Ks}$ ratios (Additional file 2: Table S7), implying that they were relatively functionally stable over recent years and may function as good targets for dosage balance selection [20]. Besides, their expression profiles in pear were tissue/development-stage/cultivar-specific (Fig. 3 \& S3), which was similar to the report on SIPG family genes [6].

Twenty-eight PbrPGs were expressed during 'Housui' pear storage, which could be divided into five groups based on their expression patterns (Fig. 4b). Similarly, 16 out of 45 PpPG genes identified from peach genome were transcribed during ripening, with diverse expression profiles [8]; of these, PpPG15, 21 and 22 might play a critical role in fruit softening [8]. In combination of the results from correlation and phylogenetic analysis, PbrPG6 might play a key role in the softening process of pear fruit (Fig. 1a \& 4c). Silence of PbrPG6 expression suppressed PG activity and maintained fruit firmness when compared with that of the control (Fig. 5). Previously, Quesada et al. [29] found that antisense of a strawberry FaPG1 gene inhibited the softening process of the ripened fruit, which might be due to a decrease in pectin solubilization and an enhancement of the amount of pectin covalently bound to the cell wall. Similar phenomenon was also observed by downregulating PG1 expression in 'Royal Gala' apple [30]. These results implied that a higher firmness in PbrPG6-silenced fruit might be due to more pectin covalently bound to the cell wall, in comparison with that of the control.

Pear, a climacteric fruit, is characterized by an increase in the respiration rate, which was associated with the accumulation of ethylene, upon initiation of ripening (Trinchero et al., 2004). In consistent with this, both ethylene evolution and respiration rate accumulated with the highest level at 18th d during fruit storage (Additional file 1: Figure S4). Ethylene plays a key role in the quality alternation during climacteric fruit ripening [31]. Mutation of an ethylene receptor, Never-ripe (Nr), inhibited the ripening process and quality formation of fruit [11]. In this study, the expression pattern of PbrPG6 mRNAs and ethylene evolution during pear storage was similar (Fig. 4b \& Additional file 1: Figure S4a); meanwhile, the impact of $1-\mathrm{MCP}$ and ethrel treatments on PbrPG6 mRNAs, PG activity and cortex firmness was opposite (Fig. 6). These results implied that the expression of PbrPG6 might be under the control of ethylene.

As final response gene in the ethylene signaling pathway, ERF could bind to the promoters of several genes, such as $A C O, P M E$, and $P G$, regulating ethylene formation and quality alternation $[12,13]$; meanwhile, the impact of ERFs on the ripening were diverse [10]. Based on bioinformatic analysis, GCC-box, which ERF could bind to [12], was observed in the promoters of PbrPG6 (Additional file 2: Table S8), suggesting that PbrERF might regulate the expression of PbrPG6. During 'Housui' pear storage, 100 PbrERFs were expressed, with diverse expression patterns (Fig. 7a); of these, 33 members illustrated relatively high correlation coefficients $(>70 \%)$ with PbrPG6 (Fig. 7b).

\section{Conclusions}

Sixty-one PbrPGs, which could be divided into six groups (A-F), were identified from pear genomes with different chromosome locations, gene structures, motifs and cis-acting elements. Most genes were derived from WGD/segmental duplication with purifying selection as 
the main driving force. Their expression profiles in pear were tissue/development-stage/cultivar-specific. During 'Housui' pear storage, in association with quality (such as color, weight loss, decay rate, firmness) alternation as well as the accumulation of PG activity, 28 PbrPG genes were transcribed, which could be classified into five categories based on different expression patterns. Of these, PbrPG6 played an important role in fruit softening in combination of bioinformatic analysis \& experimental validation. Further study found that its expression might be regulated by ethylene; and several PbrERFs might be involved in this process.

\section{Methods}

\section{Sequence retrial and annotation of PGs from pear}

Protein sequences of PGs from peach and Arabidopsis genome were downloaded from Genome Database for Rosaceae (GDR) (http://www.rosaceae.org/) [32] and The Arabidopsis Information Resource (TAIR) (http:// www.arabidopsis.org/) [33], respectively (Additional file 2: Table S9). These sequences were used as queries to perform BLASTP against the pear genome database (http://peargenome.njau.edu.cn/) [28]. Subsequently, the seed alignment file for PG domain (PF00295) which was accessed from Pfam database (http://pfam.sanger.ac.uk/) was used to build a HMM file prior to HMM searches against the local protein database of pear, using HMMER3 [20]. All candidates were then submitted to Pfam or SMART (http://smart.embl-heidelberg.de/) database to verify the presence of conserved domains, and the candidates lacking more than two highly conserved PG domains (domains I ('SPNTDGI'), II ('GDDC'), III ('CGPGHGISIGSLG'), and IV ('RIK')) were eliminated [8].

A local BLASTN against the pear EST libraries was conducted to find the records for each putative candidate with a maximum identity $>95 \%$, length $>200 \mathrm{bp}$, and E-value $<10^{-20}[20]$.

The physiological and biochemical parameters of the full-length proteins were calculated, using ProtParam tool (http://web.expasy.org/protparam/) [15]. Signal peptide and subcellular localization of each member were analyzed by SignalP 4.1 (http:/www.cbs.dtu.dk/services/ SignalP/) [34] and CELLO v2.5 server (http://cello.life. nctu.edu.tw/) [35], respectively.

\section{Phylogenetic, gene structure, motif and cis-acting element analysis}

The phylogenetic tree was constructed by MEGA 7.0.26 software, using the neighbor-joining (NJ) method with a bootstrap analysis of 1000 replicates and the poisson model [36]. Gene structures of PbrPGs obtained by alignment of open reading frames (ORFs) with the corresponding genomic sequences along with the Gene
Structure Display Server 2.0 (GSDS 2.0) program (http:// gsds.cbi.pku.edu.cn/) [15]. Motifs analysis was performed using MEME Suite 5.0.5 [37]; the identified motifs were annotated using SMART database [38]. Cis-acting regulatory elements in the $1.5 \mathrm{~kb}$ of $5^{\prime}$ regulatory region from the translational start site were identified using PlantCARE database [15]; and the divergence between upstream sequences of each paralogous gene pairs was measured by the GATA program [39], with window size set as seven and the lower cut-off score was 12 bit.

\section{Chromosomal location, synteny, and $\mathrm{Ka} / \mathrm{Ks}$ analysis}

The chromosome locations of all PbrPGs were determined according to genome annotation data, and then plotted using Circos software [40].

A method similar to that used for PGDD (http:// chibba.agtec.uga.edu/duplication/) was applied to analyze the syntenic relationship [41]; and the duplicated PbrPGs were then categorized into the following types: whole genome duplication (WGD)/segmental, tandem, singleton, proximal and dispersed. MCScanX downstream analysis tools was used to annotate the $\mathrm{Ka}$ and Ks substitution rates of the syntenic gene pair; and the KaKs Calculator 2.0 was used to determine $\mathrm{Ka}$ and Ks with the Nei-Gojobori (NG) method [42].

\section{Plant material}

Uniform and defect-free pear fruit ( $P$. pyrifolia cv. 'Housui') harvested from homogeneous trees planted in the experimental orchard of the College of Horticulture at Nanjing Agricultural University were chosen, randomly divided into several groups, packed with plastic bags, and then stored at $25^{\circ} \mathrm{C}$. Samples were taken every $6 \mathrm{~d}$ until decay rate over $20 \%$. For the sampling, the cortex from five fruit per replicate was quickly removed with a parer before analysis.

\section{Color, weight loss, decay rate, firmness, ethylene production and respiration rate determination}

Color (pericarp and first layer of the cortex tissue below pericarp), weight loss and decay rate were determined according to the method of Wang et al. [16]. A Minolta CR-400 chromameter (Konica Minolta Sensing, Inc., Osaka, Japan) was used for color analysis.

Cortex firmness was measured by Brookfield texture analyzer (CT3, Middleboro, MA, United States), using a 2-mm stainless cylindrical probe, loading at $0.5 \mathrm{~mm} \mathrm{~s}^{-1}$ in association with $10 \mathrm{~mm}$ distance.

Respiration rate was assayed by YGA2100 $\mathrm{CO}_{2}$ analyzer (Yangguangyishida Technology Co. Ltd., Beijing, China) according to the instruction of manufacturer. Ethylene evolution was determined by a GC (Agilent Technologies 7890A) fitted with flame ionization detector (FID) [43]. 


\section{Transcriptome sequencing and qRT-PCR analysis}

Total RNA was extracted using TRizol Reagents (Invitrogen, Carlsbad, CA) followed by RNase-free DNase treatment (Qiagen, Valencia, CA). RNA-seq and bioinformation analysis were conducted by Biomarker Technologies (Beijing, China). Library construction was carried out using Illumina HiSeqTM 4000 sequencing platform; pear genome database [28] was used as reference genome; FPKM was used to calculate gene expression [44]. Based on previous report, the wild P. pyrifolia was a common ancestor for $P$. pyrifolia and P. bretschneideri [45].

The primers of PbrPGs were designed, using Premier 6.0 (Additional file 2: Table S10). Total RNA was isolated using TRizol Reagents (Invitrogen, USA) followed by RNase-free DNase treatment (Qiagen, USA). Approximately $2 \mu \mathrm{g}$ of total RNA was used for first-strand cDNA synthesis using TransScript One-Step gDNA Removal and cDNA Synthesis SuperMix (TRANSGEN, China). qRT-PCR was performed according to the method of Wang et al. [15]. Pyrus Tubulin was used as the internal control, and the relative expression levels were calculated with the $2^{-\Delta \Delta \mathrm{Ct}}$ method [46].

\section{PG activity assay}

Extraction of crude PG from pear cortex and analysis of PG activity was conducted according to the instruction of manufacturer (PG-1-G, Suzhou Comin Biotechnology Co., Ltd., China). Protein concentration in crude enzyme extract was determined by protein assay kit (SSNP-1-W, Suzhou Comin Biotechnology Co., Ltd., China). The result was expressed as $\mathrm{mg}$ galacturonic acid $\mathrm{h}^{-1} \mathrm{mg}^{-1}$ protein.

\section{Transient silencing of PbrPG6 expression in pear fruit}

About $400 \mathrm{bp}$ fragment at the C-terminal of PbrPG6 were amplified before insertion into the pTRV2 vector [47]. The constructed vector and pTRV1, which could assist the movement of pTRV2 vector in cell, were transformed into A. tumefaciens strain GV3101, respectively; and then combined in a 1:1 ratio before injection into the cortex tissue [48]. The unconstructed PTRV2 vector, which was co-injected with pTRV1, was used as control. The injected fruit were then stored at $25^{\circ} \mathrm{C}$ for $5 \mathrm{~d}$ before sampling. There were three replicates per treatment with five fruit per replicate.

\section{Transient transformation of pear fruit}

Transient transformation of pear fruit was carried out following Hao et al. [12]'s method. PbrACO1 ORFs were amplified (Additional file 2: Table S10), inserted into a modified pCAMBIA 1300 vector, and then transformed into $A$. tumefaciens strain GV3101. After incubation, the suspension was centrifuged, resuspended with the infiltration buffer, and slowly injected into pear cortex before storage at $25{ }^{\circ} \mathrm{C}$. Pear fruit infiltrated with the empty vector were used as control. There were three replicates with five fruit each for each vector.

\section{Statistical analysis}

Data presented were the mean values of at least three biological replicates except for transcriptome analysis of pear fruit during storage (one replicate). SAS version 9.3 (SAS Institute, Gary, NC) was used for data analysis, using analysis of variance (PROC ANOVA) with multicomparison correction. Mean separation was determined by Duncan's multiple range test at the 0.05 level. Spearman's correlation analysis was performed to evaluate the association among attributes, which was visualized as a heatmap.

\section{Availability of supporting data}

The transcriptome clean raw reads data that support the findings of this study have been submitted to NCBI Sequence Read Archive (SRA) under Accession SUB6578158, Bioproject: PRJNA590622. All data generated or analyzed during this study are included in this published article and its supplementary information files.

\section{Supplementary information}

Supplementary information accompanies this paper at https://doi.org/10. 1186/s12870-019-2168-1.

\footnotetext{
Additional file 1: Figure S1. Multiple sequence alignment of PbrPGs. The red wireframe parts indicated four typical conserved domains of PbrPGs, which were named as domain I, II, III and IV, respectively. Figure S2. Comparative analysis of the $1.5 \mathrm{~kb}$ upstream of paralogous gene pairs. Divergence between upstream sequences of each paralogous gene pairs was measured by the GATA program (Nix and Eisen, 2005), with window size set as seven and lower cutoff score 12 bit. Solid dark lines connect similar regions and red broken lines connect matched regions in reversed orientation. Figure S3. Expression profiles of PbrPGs during development of different pear fruit. 'Housui' (a), 'Kuerlexiangli' (b), 'Nanguo' (c), 'Starkrimson' (d), 'Yali' (e) fruit were harvested from a commercial field at five developmental stages, including fruit-setting stage (period 1), physiological fruit dropping stage (period 2), fruit rapid enlargement stage (period 3), a month after fruit enlargement stage (period 4), and commercially mature stage (period 5). Data adapted from Zhang et al. (2015). Color scale at the top represented log2 transformed $($ FPKM + 1). Green indicated low level, black indicated a medium level, and red indicated high level. Figure S4. Dynamic changes of ethylene evolution and respiration rate during 'Housui' pear storage. Uniform and defect-free 'Housui' pear fruit were selected, randomly divided into several groups, packed with plastic bags, and then stored at $25^{\circ} \mathrm{C}$. Samples were taken every 6 d until decay rate over 20\%. Data represented the mean value \pm SE of three biological replicates. Different small letters with the same treatment mean significant difference among samples $(p<$ 0.05). Figure S5. qRT-PCR validation of the expression patterns of genes based on transcriptome analysis. Uniform and defect-free 'Housui' pear fruit were selected, randomly divided into several groups, packed with plastic bags, and then stored at $25^{\circ} \mathrm{C}$. Samples were taken every $6 \mathrm{~d}$ until decay rate over 20\%. Data represented the mean \pm SE of three biological replicates for qRT-PCR analysis. The expression level of PbrPG1 and Pbr5ERF39 at Oth $d$ was set as 1.0. Different small letters with the same treatment mean significant difference among samples $(p<0.05)$. Figure S6. Impact of 1-MCP and ethrel treatments on cortex firmness during
} 
pear storage. 'Housui' pears were harvested from an experimental orchard in Nanjing in 2017, and then divided into three treatments: (1) fumigated with $1.5 \mu \mathrm{L} \mathrm{L}-1$ 1-MCP for $24 \mathrm{~h}$, (2) dipped in $0.5 \mathrm{~mL} \mathrm{~L}^{-1}$ ethrel for $5 \mathrm{~min}$, and (3) dipped in $0.5 \mathrm{~mL} \mathrm{~L}^{-1} \mathrm{H}_{2} \mathrm{O}$ for $5 \mathrm{~min}$ (control). After treatments, fruits were packed with plastic bags and stored at $25^{\circ} \mathrm{C}$. Samples were taken every $6 \mathrm{~d}$. Data represent the mean $\pm \mathrm{SE}$ of three biological replicates. Different lowercase letters with the same treatment mean significant difference among samples, and different capital letters in the same sampling data mean significance among treatments $(p<0.05)$. Figure $\mathbf{S 7}$. Impact of transient overexpression of PbrACO1 on PbrPG6 mRNAs and firmness of pear fruit. (a) Expression profile of PbrACO1 in samples. (b) Impact of transient on PbrPG6 mRNAs. (c) Impact of overexpression of PbraCO1 on cortex firmness. 'Housui' fruit infiltrated with the empty vector was used as control. Data represented the mean \pm SE of three biological replicates. Different lowercase letters meant significance between samples $(p<0.05)$. The expression level of PbrACO1/PbrPG6 in control fruit was set as 1.0. Vertical bars labeled with different small letters indicated significant difference between samples at $p<0.05$ level using Duncan's multiple range test.

Additional file 2: Table S1. Information on 61 PbrPGs from pear genome. Table S2. ESTs for putative PbrPGs. Table S3. Gene features of $P G$ family genes from pear. Table S4. Cis-acting regulatory elements identified in the promoters of PbrPGs. Table S5. Motif sequences identified by MEME tools in PbrPGs. Table S6. Duplication types of PbrPG genes in pear genome. Table S7. Ka/Ks ratios of paralogous genes in PbrPG gene family. Table S8. Promoters analysis of PbrPG6 using PLACE Web Signal Scan. Table S9. Information of $P G$ genes from Arabidopsis and peach. Table S10. Primers used in this study. (XLS $667 \mathrm{~kb}$ )

\section{Abbreviations}

1-MCP: 1-Methylcyclopropene; ACO: Acid oxidase; DAFB: Days after full bloom; EMSA: Electrophoretic mobility shift assay; ERF: Ethylene response factors; ESTs: Expressed sequence tags; FID: Flame ionization detector; FPKM: Fragments per kilobase million; GDR: Genome database for Rosaceae; GRAVY: Grand average of hydropathicity; GSDS: Gene structure display server; HMM: Hidden markov model; MEME: Multiple EM for motif elicitation; MYA: Million years ago; NG: Nei-Gojobori; NJ: Neighbor-joining; Nr: Neverripe; ORFs: Open reading frames; PE: Pectinesterase; PG: Polygalacturonase; PGDD: Plant genome duplication database; PI: Isoelectric point; PME: Pectin methylesterase; TAIR: The arabidopsis information resource; WGD: Whole genome duplication; XET: Xyloglucan endo-transglycosylase; $\beta$-Gal: $\beta$ galactosidase

\section{Acknowledgments}

We thank Chao Gu, Guoming Wang and Zhihua Guo for the help in the construction of the silencing vector.

\section{Authors' contributions}

LW \& ShZ designed the experiment; LW, SuZ \& MM wrote the manuscript; MM, SuZ, ZZ, ZL, JF, \& MQ conducted the experiment; SuZ, MM, \& WL analyzed the data. $\mathrm{HZ}$ conducted the complementary experiment, analyzed the data, and improved the language of the revised manuscript. All Authors read and approved the manuscript.

\section{Funding}

This work was supported by the National Natural Science Foundation of China (31701868 and 31830081), China Postdoctoral Science Foundation (2018T110508 and 2017 M620213), and Fundamental Research Funds for the Central Universities (KUQN201813). The funding bodies only provide the financial support in the design of the study and collection, analysis, and interpretation of data and in writing the manuscript.

\section{Availability of data and materials}

Not applicable.

Ethics approval and consent to participate

Not applicable.

\section{Consent for publication}

Not applicable.

\section{Competing interests}

The authors declare that they have no competing interests.

\section{Author details}

${ }^{1}$ Centre of Pear Engineering Technology Research, State Key Laboratory of Crop Genetics and Germplasm Enhancement, Nanjing Agricultural University, Nanjing 210095, Jiangsu, China. ${ }^{2}$ USDA, ARS, U.S. Horticultural Research Laboratory, 2001 S. Rock Road, Fort Pierce, FL 34945, USA.

\section{Received: 26 September 2019 Accepted: 27 November 2019}

\section{Published online: 27 December 2019}

\section{References}

1. Barry CS, Giovannoni JJ. Ethylene and fruit ripening. J Plant Growth Regul. 2007:26(2):143-59.

2. Predieri S, Gatti E. Effects of cold storage and shelf-life on sensory quality and consumer acceptance of 'abate Fetel' pears. Postharvest Biol Tec. 2009; 51(3):342-8.

3. Brummell DA, Cin VD, Crisosto $\mathrm{CH}$, et al. Cell wall metabolism during maturation, ripening and senescence of peach fruit. J Exp Bot. 2004;55(405): 2029-39.

4. Liang Y, Yu Y, Shen X, Dong H, Lyu M, Xu L, et al. Dissecting the complex molecular evolution and expression of polygalacturonase gene family in Brassica rapa ssp. chinensis. Pant Mol Biol. 2015;89(6):629-46.

5. Sitrit Y, Bennett K, Bennett A, Bradford K, Downie A. Expression of a polygalacturonase associated with tomato seed germination. Plant Physiol. 1999;121(2):419-28.

6. Ke X, Wang H, Li Y, Zhu B, Zang Y, He Y, et al. Genome-wide identification and analysis of polygalacturonase genes in Solanum lycopersicum. Int J Mol Sci. 2018;19(8):2290.

7. Dautt-Castro M, López-Virgen AG, Ochoa-Leyva A, Contreras-Vergara CA, Sortillón-Sortillón AP, Martínez-Téllez MA, et al. Genome-wide identification of mango (Mangifera indica L.) Polygalacturonases: expression analysis of family members and Total enzyme activity during fruit ripening. Front. Plant Sci. 2019;10:969

8. Qian M, Zhang Y, Yan X, Han M, Li J, Li F, et al. Identification and expression analysis of polygalacturonase family members during peach fruit softening. Int J Mol Sci. 2016;17(11):1933.

9. Yang ZL, Liu HJ, Wang XR, Zeng QY. Molecular evolution and expression divergence of the Populus polygalacturonase supergene family shed light on the evolution of increasingly complex organs in plants. New Phytol. 2013;197(4):1353-65

10. Wang $X$, Zeng $W$, Ding $Y$, Wang $Y, N i u L$, Yao JL, et al. Peach ethylene response factor PpeERF2 represses the expression of ABA biosynthesis and cell wall degradation genes during fruit ripening. Plant Sci. 2019;283:116-26.

11. Alba R, Payton P, Fei Z, McQuinn R, Debbie P, Martin GB, et al. Transcriptome and selected metabolite analyses reveal multiple points of ethylene control during tomato fruit development. Plant Cell. 2005;17(11): 2954-65.

12. Hao PP, Wang GM, Cheng HY, Ke YQ, Zhang SL. Transcriptome analysis unravels an ethylene response factor involved in regulating fruit ripening in pear. Physiol Plantarum. 2017;163(1):124-35.

13. Fu CC, Han YC, Qi XY, Shan W, Chen JY, Lu WJ, et al. Papaya CpERF9 acts as a transcriptional repressor of cell-wall-modifying genes CPPME1/2 and CpPG5 involved in fruit ripening. Plant Cell Rep. 2016;35(11):2341-52.

14. Chen JL, Yan SJ, Feng ZS, Xiao LX, Hu XS. Changes in the volatile compounds and chemical and physical properties of Yali pear (Pyrus bertschneideri Reld) during storage. Food Chem. 2006;97(2):248-55.

15. Wang L, Wang L, Zhang Z, Ma M, Wang R, Qian M, et al. Genome-wide identification and comparative analysis of the superoxide dismutase gene family in pear and their functions during fruit ripening. Postharvest Biol Tec. 2018;143:68-77.

16. Wang L, Ma M, Zhang Y, Wu Z, Guo L, Luo W, et al. Characterization of the genes involved in malic acid metabolism from pear fruit and their expression profile after postharvest 1-MCP/ethrel treatment. J Agr Food Chem. 2018;66:8772-82.

17. Li J, Di T, Bai J. Distribution of volatile compounds in different fruit structures in four tomato cultivars. Molecules. 2019;24(14):2594.

18. Li J, Yan J, Ritenour MA, Wang J, Cao J, Jiang W. Effects of 1methylcyclopropene on the physiological response of Yali pears to bruise damage. Sci Hortic. 2016;200:137-42. 
19. Zhou R, Li Y, Yan L, Xie J. Effect of edible coatings on enzymes, cellmembrane integrity, and cell-wall constituents in relation to brittleness and firmness of Huanghua pears (Pyrus pyrifolia Nakai, cv. Huanghua) during storage. Food Chem. 2011;124(2):569-75.

20. Qiao X, Li M, Li L, Yin H, Wu J, Zhang S. Genome-wide identification and comparative analysis of the heat shock transcription factor family in Chinese white pear (Pyrus bretschneideri) and five other Rosaceae species. BMC Plant Bio. 2015;15(1):12.

21. Zhang MY, Xue C, Xu L, Sun H, Qin MF, Zhang S, et al. Distinct transcriptome profiles reveal gene expression patterns during fruit development and maturation in five main cultivated species of pear (Pyrus L.). Sci Rep. 2016;6:28130

22. Hao D, Ohme-Takagi M, Sarai A. Unique mode of GCC box recognition by the DNA-binding domain of ethylene-responsive element-binding factor (ERF domain) in plant. J Biol Chem. 1998;273(41):26857-61.

23. Li $X$, Tao S, Wei S, Ming M, Huang $X$, Zhang $S$, et al. The mining and evolutionary investigation of AP2/ERF genes in pear (Pyrus). BMC Plant Bio. 2018;18(1):46.

24. Zhu M, Shen W, Zhou P. Research advances on mechanism of fruit ripening and softening. Mol Plant Breed. 2005;3(3):421-6.

25. Chen H, Shao H, Fan S, Ma J, Zhang D, Han M. Identification and phylogenetic analysis of the POLYGALACTURONASE gene family in apple. Horti Plant J. 2016;2(5):241-52.

26. Torki M, Mandaron P, Mache R, Falconet D. Characterization of a ubiquitous expressed gene family encoding polygalacturonase in arabidopsis thaliana. Gene. 2000;242(1-2):427-36.

27. Stratilová E, Mislovičová D, Kačuráková M, Machová E, Kolarová N, Markovič $\mathrm{O}$, et al. The glycoprotein character of multiple forms of aspergillus polygalacturonase. J Protein Chem. 1998;17(2):173-9.

28. Wu J, Wang Z, Shi Z, Zhang S, Ming R, Zhu S, et al. The genome of the pear (Pyrus bretschneideri Rehd.). Genome Res. 2013;23(2):396-408.

29. Quesada MA, Blanco-Portales R, Posé S, García-Gago JA, Jiménez-Bermúdez S, Muñoz-Serrano A, et al. Antisense down-regulation of the FaPG1 gene reveals an unexpected central role for polygalacturonase in strawberry fruit softening. Plant Physiol. 2009;150(2):1022-32.

30. Atkinson RG, Sutherland PW, Johnston SL, Gunaseelan K, Hallett IC, Mitra D, et al. Down-regulation of POLYGALACTURONASE1 alters firmness, tensile strength and water loss in apple (Malus $\mathrm{x}$ domestica) fruit. BMC Plant Bio. 2012;12(1):129.

31. Wang L, Baldwin EA, Bai J. Recent advance in aromatic volatile research in tomato fruit: the metabolisms and regulations. Food Bioprocess Tech. 2016; 9(2):203-16

32. Jung S, Staton M, Lee T, Blenda A, Svancara R, Abbott A, et al. GDR (genome database for Rosaceae): integrated web-database for Rosaceae genomics and genetics data. Nucleic Acids Res. 2007;36:D1034-40.

33. Swarbreck D, Wilks C, Lamesch P, Berardini TZ, Garcia-Hernandez M, Foerster $\mathrm{H}$, et al. The Arabidopsis information resource (TAIR): gene structure and function annotation. Nucleic Acids Res. 2007;36:D1009-14.

34. Petersen TN, Brunak S, Von Heijne G, Nielsen H. SignalP 4.0: discriminating signal peptides from transmembrane regions. Nat. Methods. 2011;8(10):785-6.

35. Dong Y, Li C, Zhang Y, He Q, Daud MK, Chen J, et al. Glutathione Stransferase gene family in Gossypium raimondii and G. arboreum: comparative genomic study and their expression under salt stress. Front Plant Sci. 2016;7:139.

36. Kumar S, Nei M, Dudley J, Tamura K. MEGA: a biologist-centric software for evolutionary analysis of DNA and protein sequences. Brief Bioinform. 2008 9(4):299-306.

37. Bailey TL, Johnson J, Grant CE, Noble WS. The MEME suite. Nucleic Acids Res. 2015;43:W39-49.

38. Letunic I, Doerks T, Bork P. SMART 7: recent updates to the protein domain annotation resource. Nucleic Acids Res. 2011;40:D302-5.

39. Nix DA, Eisen MB. GATA: a graphic alignment tool for comparative sequence analysis. BMC Bioinformatics. 2005;6(1):9.

40. Krzywinski M, Schein J, Birol I, Connors J, Gascoyne R, Horsman D, et al. Circos: an information aesthetic for comparative genomics. Genome Res. 2009;19(9):1639-45.

41. Lee $T H$, Tang H, Wang $X$, Paterson AH. PGDD: a database of gene and genome duplication in plants. Nucleic Acids Res. 2012;41:D1152-8.

42. Wang D, Zhang Y, Zhang Z, Zhu J, Yu J. KaKs_Calculator 2.0: a toolkit incorporating gamma-series methods and sliding window strategies. Genom Proteom. 2010;8(1):77-80.
43. Cai H, An X, Shuai H, Li J, Yu M, Ma R, et al. Effect of 1-MCP on the production of volatiles and biosynthesis-related gene expression in peach fruit during cold storage. Postharvest Bio Tec. 2018;141:50-7.

44. Trapnell C, Williams BA, Pertea G, Mortazavi A, Kwan G, Van Baren MJ, et al. Transcript assembly and quantification by RNA-Seq reveals unannotated transcripts and isoform switching during cell differentiation. Nat Biotechnol. 2010;28(5):511-5.

45. Wu J, Wang Y, Xu J, Korban SS, Fei Z, Tao S, et al. Diversification and independent domestication of Asian and European pears. Genome Biol. 2018;19(1):77.

46. Livak KJ, Schmittgen TD. Analysis of relative gene expression data using real-time quantitative $P C R$ and the $2^{-\Delta \Delta C T}$ method. Methods. 2001;25(4): 402-8.

47. Bai S, Tuan PA, Tatsuki M, Yaegaki H, Ohmiya A, Yamamizo C, et al. Knockdown of carotenoid cleavage dioxygenase 4 (CCD4) via virus-induced gene silencing confers yellow coloration in peach fruit: evaluation of gene function related to fruit traits. Plant Mol Biol Rep. 2016;34(1):257-64.

48. Gu C, Guo ZH, Cheng HY, Zhou YH, Qi KJ, Wang GM, et al. A HD-ZIP ॥ HOMEBOX transcription factor, PpHB. G7, mediates ethylene biosynthesis during fruit ripening in peach. Plant Sci. 2019;278:12-9.

\section{Publisher's Note}

Springer Nature remains neutral with regard to jurisdictional claims in published maps and institutional affiliations.
Ready to submit your research? Choose BMC and benefit from:

- fast, convenient online submission

- thorough peer review by experienced researchers in your field

- rapid publication on acceptance

- support for research data, including large and complex data types

- gold Open Access which fosters wider collaboration and increased citations

- maximum visibility for your research: over $100 \mathrm{M}$ website views per year

At BMC, research is always in progress.

Learn more biomedcentral.com/submissions 